\section{Patienten-Ratgeber für} Hämorrhoiden-Geplagte

Die Dr. Kade Pharmazeutische Fabrik hat den Ratgeber "Gut zu wissen“ für Patienten mit Hämorrhoiden herausgegeben. Er vermittelt mit anschaulichen Illustrationen, was sich hinter dem Begriff „Hämorrhoiden“ verbirgt, wie sie entstehen und was man tun kann, um ihnen vorzubeugen und Beschwerden zu lindern. Die Broschüre kann zur Abgabe an Patienten angefordert werden unter

Dr. Kade Pharmazeutische Fabrik, Stichwort „Patientenratgeber Hämorrhoiden",

Rigistraße 2, 12277 Berlin

Sie ist auch im Internet abrufbar unter www.posterisan.de oder

www.haemorrhoiden.de.

Nach Informationen von Dr. Kade

\section{Neuer TNF-Blocker gegen} Psoriasis-Arthritis zugelassen

Der monoklonale humane IgG1-Antikörper Glimumab (Simponi ${ }^{\circledR}$ ) ist zur Behandlung der Psoriasis-Arthritis zugelassen. Dabei handelt es sich um den ersten und bislang einzigen TNFBlocker, bei dem es genügt, ihn einmal monatlich subkutan zu injizieren. Klinische Daten belegen, dass Glimumab die radiologisch nachweisbare Progression der Erkrankung und die Entstehung struktureller Schäden effektiv verhindern kann.

Nach Informationen von Essex Pharma

\section{Neue Fortbildungsplattform sorgt für mehr Durchblick}

Rund 80.000 Veranstaltungen zur fachlichen Fort- und Weiterbildung finden Ärzte auf der Online-Plattform www.my-medical-education.com im Internet. Das Angebot reicht von kleinen Praxissymposien über mehrtägige Seminare bis hin zu großen internationalen Kongressen.

Um bei dem immensen Angebot den Überblick zu behalten, welche Fortbildungen sich wirklich lohnen, haben die Nutzer die Möglichkeit, einzelne Veranstaltungen zu bewerten.

Nach Informationen von Wefra Publishing

\title{
„Physiologisches“ Anti-Aging für die Haut ab 60
}

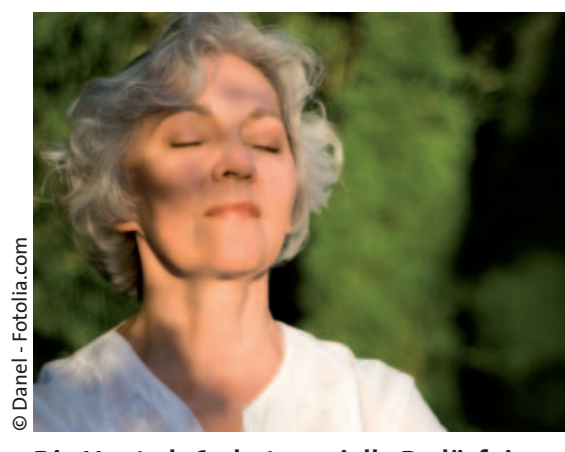

Die Haut ab 60 hat spezielle Bedürfnisse.

- Die Haut verändert sich mit zunehmendem Alter in mehrfacher Hinsicht: Der Gehalt an essenziellen Fettsäuren (insbesondere Linolsäure) nimmt ab. Auch kollagene Fasern und Hyaluronsäure sind reduziert. Seit Januar 2010 steht mit Avène Sérénage ein neues Anti-Aging-Produkt zur Verfügung, das speziell für Frauen im Alter ab 60 Jahren entwickelt worden ist. In der Creme ist Glycoleol ${ }^{\circledR}$ enthalten, eine Vorstufe der Linolsäure, das als Depot wirkt. In der Haut wird es kontinuierlich enzymatisch zu Linolsäure metabolisiert Dadurch wird der Säureschutzmantel der
Haut konstant unterstützt und der epidermale Wasserverlust vermindert. Ein weiterer Bestandteil ist H.A.F ${ }^{\circledR}$. Diese Hyaluronsäure-Fragmente sind in der Lage, tief in die Haut einzudringen. Die Fragmente induzieren auch die zelleigene Produktion von Hyaluronsäure und von Kollagen. Die Wasseraufnahmekapazität der Haut steigt, das kollagene Stützgewebe wird regeneriert und nimmt an Dichte zu. Ein weiterer Wirkstoff ist Pro-Tocopherol ${ }^{\circledR}$, die einzige stabile und leicht von der Haut assimilierbare Form von Vitamin E. Auch diese Substanz wirkt als Depot, das nach Bedarf in aktives Tocopherol umgewandelt werden kann. So entsteht ein kontinuierlicher Schutz gegen freie Radikale und damit gegen die nachteiligen Effekte von UV-Strahlen oder weiteren belastenden Umweltfaktoren auf die Haut.

Die neue Öl-in-Wasser-Emulsion enthält keine Parabene, ist hypoallergen und nicht komedogen. Sie enthält zu $52 \%$ Thermalwasser mit niedrigem Anteil an Mineralstoffen und hoher Konzentration an Kieselerde. af

Pierre Fabre Infotag, München, 5.11.2009 (Veranstalter: Pierre Fabre Dermo-Kosmetik).

\section{Ein Plus für die Compliance: Komplette Immuntherapie in nur vier Sitzungen}

— In der Behandlung der allergischen Rhinitis spielt die Auswahl der geeigneten spezifischen Immuntherapie (SIT) eine entscheidende Rolle. Denn die Eigenschaften des verordneten Medikaments beeinflussen neben Wirksamkeit und Verträglichkeit auch unmittelbar die Compliance.

Vorteile gegenüber einer Langzeit-Immuntherapie bietet hier das Präparat Pollinex ${ }^{\circledR}$ quattro, da es lediglich vier Sitzungen erfordert. Diese kurze Therapiedauer wird durch den dualen Wirkansatz von Allergoid und dem Adjuvans $M P L^{\circledR}$ ermöglicht. Dadurch wird die TH-1-Immunantwort verstärkt, wie Dr. Jens Vollmar, München, erläuterte.

Die Effektivität dieser Behandlung belegt eine Vergleichsstudie, die Dr. Dietrich Stollewerk, Köln, präsentierte: 233 Baum- und Pollenallergiker zwischen 9 und 75 Jahren wurden entweder mit einer Langzeit-SIT oder einer Kurzzeit-SIT mit vier Injektionen (Pollinex ${ }^{\circledast}$ quattro) behandelt. Die Studienteilnehmer durften in den drei vorangegangenen Jahren keine SIT erhalten haben. Ziel der deskriptiven Studie war, die Äquivalenz beider Methoden nachzuweisen.

Die Ergebnisse zeigten, dass die KurzzeitSIT der Langzeit-SIT weder bei der Augennoch bezüglich der Nasensymptomatik unterlegen war. Die Nebenwirkungen beschränkten sich bei der Kurzzeittherapie auf lokale Ereignisse. Stollewerk hält daher die komplette Immuntherapie mit lediglich vier Injektionen bezüglich Compliance und Kosten einer Langzeittherapie für überlegen.

slx

Satellitensymposium „Komplett und flexibel: Die Immuntherapie mit 4 Injektionen. Wie viel SIT kommt beim Patienten an?", Berlin, 5.9.2009 (Veranstalter: Bencard Allergie). 